\title{
Noether symmetry approach in Eddington-inspired Born-Infeld gravity
}

\author{
Thanyagamon Kanesom ${ }^{1, a}$, Phongpichit Channuie ${ }^{2,3,4, b}$, Narakorn Kaewkhao ${ }^{1, \mathrm{c}}$ \\ ${ }^{1}$ Division of Physical Science (Physics), Faculty of Science, Prince of Songkla University, Hatyai 90112, Thailand \\ ${ }^{2}$ School of Science, Walailak University, Nakhon Si Thammarat 80160, Thailand \\ ${ }^{3}$ College of Graduate Studies, Walailak University, Nakhon Si Thammarat 80160, Thailand \\ ${ }^{4}$ Research Group in Applied, Computational and Theoretical Science (ACTS), Walailak University, Nakhon Si Thammarat 80160, Thailand
}

Received: 21 February 2021 / Accepted: 14 April 2021 / Published online: 26 April 2021

(C) The Author(s) 2021

\begin{abstract}
In this work, we take a short recap of a formal framework of the Eddington-inspired Born-Infeld (EiBI) theory of gravity and derive the point-like Lagrangian for underlying theory based on the use of Noether gauge symmetries (NGS). We study a Hessian matrix and quantify EulerLagrange equations of EiBI universe. We discuss the NGS approach for the Eddington-inspired Born-Infeld theory and show that there exists the de Sitter solution in this gravity model.
\end{abstract}

\section{Contents}

1 Introduction ................. 1

2 Eddington-inspired Born-Infeld gravity . . . . . . 2

3 Hessian matrix and Euler-Lagrange equations of EiBI universe . . . . . . . . . . . . 3

4 Noether gauge symmetries in EiBI gravity . . . . . 4

5 Remarks on exact cosmological solutions . . . . . . 5

6 Conclusion .................. 7

References . . . . . . . . . . . . . . 7

\section{Introduction}

Various cosmological observations make a strong evidence that the expansion of the universe is presently accelerating. These experimental results include Type Ia Supernovae $[1,2]$, cosmic microwave background (CMB) radiation [39], large scale structure [10,11], baryon acoustic oscillations (BAO) [12] as well as weak lensing [13]. An expansion phase

\footnotetext{
a e-mail: thanyagamon1995@gmail.com

be-mail: channuie@gmail.com

c e-mail: naragorn.k@psu.ac.th (corresponding author)
}

can be basically explained by the simplest model: the socalled Lambda cold dark matter $(\Lambda \mathrm{CDM})$ [14]. However, the $\Lambda \mathrm{CDM}$ model is plagued by the cosmological problem [15] and the coincident problem [16]. There are at least two promising explanations to date to describe the late-time cosmic acceleration. The first one assumes the introduction of the so-called "dark energy (DE)" in the context of conventional general relativity. Another convincing approach is to engineer Einstein gravity on the large-scale methodology (see for reviews on not only dark energy problem but also modified gravity theories, e.g., [17-21]). However, the DE sector remains still unknown and possesses one of the unsolved problems in physics.

Therefore, it opens opportunities to search for modified theories of gravity to deal with such problems. By modifying the geometrical part of Einstein field equations or adding scalar field to the right-hand side of the Einstein field equations, both alternatives are able to explain effects of dark ingredients with acceptable assertions [22]. One of the simplest modifications to the standard general relativity is the $f(R)$ theories of gravity in which the Lagrangian density $f$ is an arbitrary function of the scalar curvature $R[23,24]$. Among numerous alternatives, these theories include higher order curvature invariants, see rigorous reviews on $f(R)$ theories $[25,26]$ as well as on Born-Infeld inspired modifications of gravity [27]. See also a recent review on modified gravity theories on inflation, bounce and late-time Evolution [28]. There have also been works regarding the inclusion on EiBI on a larger class of theories named Ricci-based gravity theories [29-31]. Additionally, there have been other works in EiBI theories appeared to have rigid consequences, see [32-36].

In cosmological framework, the Noether symmetry (NS) approach has revealed a useful tool not only to fix physically viable cosmological models with respect to the conserved 
quantities, but also to reduce dynamics and achieve exact solutions [37,38]. Moreover, the existence of Noether symetries plays crucial roles when studying quantum cosmology [39]. The Noether symmetry approach has been employed to various cosmological scenarios so far including the $f(T)$ gravity [40], the $f(R)$ gravity [41], the alpha-attractors [42], spherical and cylindrical solutions in $f(T)$ gravity [43], $f(G)$ gravity [44], non-local curvature and Gauss-Bonnet cosmologies [45], and others cosmological scenarios, e.g. [46-55]. The study of Palatini $f(R)$ cosmology using the NS approach for the matter-dominated universe was carried out in Ref. [56,57]. Moreover, the exact solutions for potential functions, scalar field and the scale factors in the Bianchi models have been investigated in [58,59].

Apart from the NS approach $[37,38]$, the Noether Gauge Symmetry (NGS) [60-62] is more generalized. In this work, we examine a formal framework of Eddington-inspired Born-Infeld (EiBI) gravity through the NGS approach and present a detailed calculation of the point-like Lagrangian. Notice that the point-like Lagrangian derived from the alternative form of the EiBI action was proposed by Delsate and Steindoff [63] instead of using the original form of the EiBI action suggested by Máximo Bañadoz [64].

This paper is organized as follows: we will start by making a short recap of a formal framework of the Eddingtoninspired Born-Infeld theory of gravity in Sect. 2. Here we derive the point-like Lagrangian for underlying theory. In Sect. 3, we study a Hessian matrix and quantify EulerLagrange equations of EiBI universe. In Sect. 4, the NGS approach for the Eddington-inspired Born-Infeld theory is discussed. We comment on exact cosmological solutions of the EiBI theory based on the use of Noether symmetries of point-like Lagrangians in Sect. 5. Finally, we conclude our findings in the last section.

$$
\begin{aligned}
S_{\mathrm{EiBI}}(g, \Gamma)= & \frac{2}{\kappa} \int d^{4} x\left[\sqrt{\left|g_{\mu \nu}+\kappa R_{\mu \nu}(\Gamma)\right|}-\lambda \sqrt{\left|g_{\mu \nu}\right|}\right] \\
& +S_{m}\left(g_{\mu \nu}, \Psi\right)+S_{\phi}\left(g_{\mu \nu}, \phi\right),
\end{aligned}
$$

where $\lambda=1+\kappa \Lambda$ is a dimensionless constant displaying the relation between the EiBI free parameter $\kappa$ (with a dimension of $M_{P}^{2}$ ) and the cosmological constant $\Lambda$ (with a dimension of $\left.M_{P}^{-2}\right)$; whilst $S_{m}\left(g_{\mu \nu}, \Psi\right)$ and $S_{\phi}\left(g_{\mu \nu}, \phi\right)$ represent the matter field action and the scalar field action, respectively. The authors of Refs. [65,66] have realised why these theories (including EiBI) do not propagate ghosts. This is due to their projective symmetry, which is realised by taking into account only the symmetric part of the Ricci tensor in the EiBI action. Therefore, in order to avoid ghosts, the Ricci scalar of Eq. (2.1) should actually be the symmetric part of the Ricci tensor, otherwise the theory propagates ghosts.

Throughout this work, we set $\frac{8 \pi G_{N}}{c^{4}}=1$. After performing variation of Eq. (2.1) with respect to $\Gamma_{\mu \nu}^{\lambda}$, we obtain the relation between two metric tensors, i.e. $q_{\mu \nu}=g_{\mu \nu}+\kappa R_{\mu \nu}(\Gamma)$. Hence Eq. (2.1) can be written in the bi-metric form as

$$
\begin{aligned}
S_{\mathrm{EiBI}}(g, \Gamma)= & \frac{2}{\kappa} \int d^{4} x\left[\sqrt{\left|q_{\mu \nu}\right|}-\lambda \sqrt{\left|g_{\mu \nu}\right|}\right] \\
& +S_{m}\left(g_{\mu \nu}, \Psi\right)+S_{\phi}\left(g_{\mu \nu}, \phi\right) .
\end{aligned}
$$

With the help of two ansatz forms of a spatially flat FLRW metric

$d s_{g}^{2}=g_{\mu \nu} d x^{\mu} d x^{\nu}=-N(t)^{2} d t^{2}+a(t)^{2} d \vec{x}^{2}$,
$d s_{q}^{2}=q_{\mu \nu} d x^{\mu} d x^{\nu}=-M(t)^{2} d t^{2}+b(t)^{2} d \vec{x}^{2}$,

$d s_{q}^{2}=q_{\mu \nu} d x^{\mu} d x^{\nu}=-M(t)^{2} d t^{2}+b(t)^{2} d \vec{x}^{2}$,

the EiBI action (2.2) can be expressed in terms of the cosmological variables as follows:

$$
\begin{aligned}
S_{\mathrm{EiBI}}=\frac{2}{\kappa} v_{0} \int d t \mathcal{L}_{\mathrm{EiBI}}=\frac{2 v_{0}}{\kappa} \int d t\left[\sqrt{a^{6}\left[N^{2}-3 \kappa\left(\frac{\dot{M} \dot{b}}{M b}-\frac{\ddot{b}}{b}\right)\right]\left[1+\frac{\kappa}{a^{2} M^{2}}\left(-\ddot{b} b+2 \dot{b}^{2}-\frac{b \dot{b} \dot{M}}{M}\right)\right]^{3}}\right. \\
\left.-N a^{3}(1+\kappa \Lambda)\right]-N a^{3}\left[-\rho_{m}(a)+\frac{l \dot{\phi}^{2}}{N^{2}}-2 V(\phi)\right]
\end{aligned}
$$

\section{Eddington-inspired Born-Infeld gravity}

In 2009, Máximo Bañadoz [64] proposed a new form of the Born-Infeld action under Palatini formalism. This is the socalled Eddington-inspired-Born-Infeld (EiBI) gravity. This action is written as follows: where $v_{0}$ is the spatial volume obtaining after a proper compactification for spatial flat section. $l=+1$ and $l=-1$ denote an ordinary scalar field and phantom scalar field, respectively. It is probably impossible to get rid of all second order derivative in Eq. (2.5) by performing an integration by parts. Therefore we neglect to write an explicit form of the point-like Lagrangian. In 2012, Delsate and Steindoff [63], however, wisely proposed the bi-metric form of the EiBI action under the metric formulation written as 
$S_{\mathrm{EiBI}}(g, q)=\lambda \int d^{4} x \sqrt{-q}\left[R(q)-\frac{2 \lambda}{\kappa}+\frac{1}{\kappa}\left(q^{\alpha \beta} g_{\alpha \beta}-2 \sqrt{\frac{g}{q}}\right)\right]$

$$
+S_{\phi}(g, \phi)+S_{M}(g, \Phi) \text {. }
$$

Notice that only $g_{\mu \nu}$ interacts with matter and scalar fields whereas $q_{\mu \nu}$ defines the background metric regarded as the fundamental reference frame of the universe [67]. Because the alternative action yields identical field equations as provided in the Bañadoz action, this indicates that two action forms are equivalent. Using a relation, $S=v_{0} \int d t \mathcal{L}$ [68] and the relation between two metric tensors, $q_{\mu \nu}=g_{\mu \nu}+$ $\kappa R_{\mu \nu}(\Gamma)$, the point-like Lagrangian can be extracted from equation (2.6) as follows

$$
\begin{aligned}
\mathcal{L}_{\mathrm{EiBI}}= & \lambda M b^{3}\left[-\frac{6 \dot{b}^{2}}{M^{2} b^{2}}-\frac{2 \lambda}{\kappa}+\frac{1}{\kappa}\left(\frac{N^{2}}{M^{2}}+3 \frac{a^{2}}{b^{2}}\right)\right] \\
& +N a^{3}\left(l \frac{\dot{\phi}^{2}}{N^{2}}-2 V(\phi)-2 \rho_{m}(a)\right) .
\end{aligned}
$$

Here the number of configuration space (or the minisuperspace) variables equal to five due to the appearance of variables $\{a(t), b(t), M(t), N(t), \phi(t)\}$ in Eq. (2.7). Apart from the kinetic part of the Lagrangian, we can set $V_{\text {eff }}=$ $\frac{\lambda M b^{3}}{\kappa}\left[2 \lambda-\left(\frac{N^{2}}{M^{2}}+3 \frac{a^{2}}{b^{2}}\right)\right]+2 N a^{3}\left(V(\phi)+\rho_{m}(a)\right)$ as an effective potential in the gravity model. It also notes that Eq. (2.7) is a singular Lagrangian due to the existence of two Lapse functions, $N(t)$ and $M(t)$ as shown in the denominators of Eq. (2.7).

\section{Hessian matrix and Euler-Lagrange equations of EiBI universe}

In the absent of $\{\dot{a}, \dot{M}, \dot{N}\}$ in the EiBI point-like Lagrangian, the EiBI Hessian matrix can be written as

$$
\begin{aligned}
{\left[W_{i j}\right]_{\text {EiBI }}=} & {\left[\begin{array}{ccccc}
\frac{\partial^{2} L}{\partial \dot{a}^{2}} & \frac{\partial^{2} L}{\partial \dot{a} \partial \dot{b}} & \frac{\partial^{2} L}{\partial \dot{a} \partial \dot{N}} & \frac{\partial^{2} L}{\partial \dot{a} \partial \dot{M}} & \frac{\partial^{2} L}{\partial \dot{a} \partial \dot{\phi}} \\
\frac{\partial^{2} L}{\partial \dot{b} \partial \dot{a}} & \frac{\partial^{2} L}{\partial \dot{b}^{2}} & \frac{\partial^{2} L}{\partial \dot{b} \partial \dot{N}} & \frac{\partial^{2} L}{\partial \dot{b} \partial \dot{M}} & \frac{\partial^{2} L}{\partial \dot{b} \partial \dot{\phi}} \\
\frac{\partial^{2} L}{\partial \dot{N} \partial \dot{a}} & \frac{\partial^{2} L}{\partial \dot{N} \partial \dot{b}} & \frac{\partial^{2} L}{\partial \dot{N}^{2}} & \frac{\partial^{2} L}{\partial \dot{N} \partial \dot{M}} & \frac{\partial^{2} L}{\partial \dot{N} \partial \dot{\phi}} \\
\frac{\partial^{2} L}{\partial \dot{M} \partial \dot{a}} & \frac{\partial^{2} L}{\partial \dot{M} \partial \dot{b}} & \frac{\partial^{2} L}{\partial \dot{M} \partial \dot{N}} & \frac{\partial^{2} L}{\partial \dot{M}^{2}} & \frac{\partial^{2} L}{\partial \dot{M} \partial \dot{\phi}} \\
\frac{\partial^{2} L}{\partial \dot{\phi} \partial \dot{a}} & \frac{\partial^{2} L}{\partial \dot{\phi} \partial \dot{b}} & \frac{\partial^{2} L}{\partial \dot{\phi} \partial \dot{N}} & \frac{\partial^{2} L}{\partial \dot{\phi} \partial \dot{M}} & \frac{\partial^{2} L}{\partial \dot{\phi}^{2}}
\end{array}\right] } \\
= & {\left[\begin{array}{ccccc}
0 & 0 & 0 & 0 & 0 \\
0-\frac{12 \lambda b}{M} & 0 & 0 & 0 \\
0 & 0 & 0 & 0 & 0 \\
0 & 0 & 0 & 0 & 0 \\
0 & 0 & 0 & 0 & \frac{2 a^{3} l}{N}
\end{array}\right] . }
\end{aligned}
$$

Clearly, the determinant of the Hessian matrix of the EiBI point-like Lagrangian equals zero indicates again that Eq. (2.7) is a singular Lagrangian. Accordingly, variables $\{a, N, M\}$ do not contribute to dynamics and have to be considered as a further constraint equations. This tells us that $a(t), M(t)$ and $N(t)$ are not independent variables anymore then we can set them to an arbitrary functions of time [69], i.e. $\mathcal{F}(t)=\mathcal{F}(a, M, N)$. Variables $b(t)$ and $\phi(t)$ however remain considered independently. With the definition of the Euler-Lagrange equations, we can show that [70]

$$
\begin{aligned}
\frac{d}{d t} \frac{\partial \mathcal{L}}{\partial \dot{q}^{i}}-\frac{\partial \mathcal{L}}{\partial q^{i}} & =\frac{\partial}{\partial q^{j}}\left(\frac{\partial \mathcal{L}}{\partial \dot{q}^{i}}\right) \frac{d q^{j}}{d t}+\frac{\partial}{\partial \dot{q}^{j}}\left(\frac{\partial \mathcal{L}}{\partial \dot{q}^{i}}\right) \frac{d \dot{q}^{j}}{d t}-\frac{\partial \mathcal{L}}{\partial q^{i}}, \\
& =\ddot{q}^{j} \frac{\partial^{2} \mathcal{L}}{\partial \dot{q}^{j} \partial \dot{q}^{i}}+\dot{q}^{j} \frac{\partial^{2} \mathcal{L}}{\partial q^{j} \partial \dot{q}^{i}}-\frac{\partial \mathcal{L}}{\partial q^{i}}=0 \\
\ddot{q}^{j} W_{i j}=\ddot{q}^{j} \frac{\partial^{2} \mathcal{L}}{\partial \dot{q}^{j} \partial \dot{q}^{i}} & =-\dot{q}^{j} \frac{\partial^{2} \mathcal{L}}{\partial q^{j} \partial \dot{q}^{i}}+\frac{\partial \mathcal{L}}{\partial q^{i}}
\end{aligned}
$$

Here the configuration space variables are $q_{i}=\{a, b, M, N, \phi\}$ and their time derivative on the tangent space are $\dot{q}_{i}=$ $\frac{d q_{i}}{d t}=\{\dot{a}, \dot{b}, \dot{M}, \dot{N}, \dot{\phi}\}$. Because $\frac{\partial^{2} \mathcal{L}}{\partial \dot{q}^{j} \partial \dot{q}^{i}}=0$ and $\frac{\partial^{2} \mathcal{L}}{\partial q^{j} \partial \dot{q}^{i}}=0$ for variables $\{a, M, N\}$ in EiBI gravity, the Euler-Lagrange equations of these variables can be reduced to $\frac{\partial \mathcal{L}}{\partial a}=\frac{\partial \mathcal{L}}{\partial M}=$ $\frac{\partial \mathcal{L}}{\partial N}=0$ where $\dot{a}, \dot{M}, \dot{N}$ and $\ddot{a}, \ddot{M}, \ddot{N}$ can be set arbitrarily [70]. As expected, $\ddot{b}$ and $\ddot{\phi}$ are determined from taking variation Lagrangian with respect to the dynamical variables and their time derivative as shown on the right-hand side of Eq. (3.3). We have to keep in mind that a crucial concept of a gauge theory is the general solution of the equations of motion which contains arbitrary functions of time and the canonical variables are not all independent but relate among each others via the constraint equations [70]. As the results of the vanishing of $\left\{\frac{\partial^{2} \mathcal{L}}{\partial \dot{a}^{2}}=\frac{\partial^{2} \mathcal{L}}{\partial \dot{M}^{2}}=\frac{\partial^{2} \mathcal{L}}{\partial \dot{N}^{2}}=0\right\}$, the canonical momenta associated to $a, N$, and $M$ yield $p_{a}=\partial \mathcal{L} / \partial \dot{a}=0, p_{N}=\partial \mathcal{L} / \partial \dot{N}=0, p_{M}=\partial \mathcal{L} / \partial \dot{M}=$ 0 , respectively. The Hamiltonian constraint equation can be straightforwardly derived from the canonical momenta via the Lagrangian and theirs Lagrange multipliers $\left\{\lambda_{i}=\right.$ $\left.\lambda_{a}(t), \lambda_{M}(t), \lambda_{N}(t)\right\}$ as follows:

$$
\begin{aligned}
\mathcal{H}_{\mathrm{EiBI}}= & \frac{\partial \mathcal{L}_{\mathrm{EiBI}}}{\partial \dot{q}_{i}} \dot{q}_{i}-\mathcal{L}_{\mathrm{EiBI}}=\frac{\partial \mathcal{L}_{\mathrm{EiBI}}}{\partial \dot{b}} \dot{b}+\frac{\partial \mathcal{L}_{\mathrm{EiBI}}}{\partial \dot{\phi}} \dot{\phi}-\mathcal{L}_{\mathrm{EiBI}}, \\
\mathcal{H}_{\mathrm{EiBI}, \text { tot }}= & \mathcal{H}_{\mathrm{EiBI}}+\Sigma \lambda_{i} p_{i}, \\
= & \left(-\frac{12 \lambda b \dot{b}}{M}\right) \dot{b}+\left(\frac{2 a^{3} l \dot{\phi}}{N}\right) \dot{\phi}-\mathcal{L}_{\mathrm{EiBI}}+\lambda_{a} p_{a} \\
& +\lambda_{N} p_{N}+\lambda_{M} p_{M}=0, \\
= & {\left[-\frac{6 \lambda b \dot{b}^{2}}{M}+\frac{a^{3} l \dot{\phi}^{2}}{N}\right]+\frac{1}{\kappa}\left[2 \lambda^{2} M b^{3}-\lambda b^{3} \frac{N^{2}}{M}-3 \lambda a^{2} M b\right.} \\
& \left.+2 \kappa N a^{3} V(\phi)+2 \kappa \rho(a) N a^{3}\right]+\lambda_{a} p_{a}+\lambda_{M} p_{M} \\
& +\lambda_{N} p_{N}=0,
\end{aligned}
$$

where the Lagrange multipliers, $\lambda_{a}(t), \lambda_{M}(t)$, and $\lambda_{N}(t)$, re arbitrary functions of time. The total EiBI Hamiltonian $\left(\mathcal{H}_{\text {EiBI }}\right)$ can be used to evaluate an evolution invoking the 
Hamiltonian equations of motion as follows:

$$
\begin{aligned}
\dot{a}= & \frac{\partial \mathcal{H}_{\mathrm{EiBI}, \text { tot }}}{\partial p_{a}}=\lambda_{a}(t), \dot{M}=\frac{\partial \mathcal{H}_{\mathrm{EiBI}, \text { tot }}}{\partial p_{M}}=\lambda_{M}(t), \\
\dot{N}= & \frac{\partial \mathcal{H}_{\mathrm{EiBI}, \text { tot }}}{\partial p_{N}}=\lambda_{N}(t), \\
\dot{b}= & \frac{\partial \mathcal{H}_{\mathrm{EiBI}, \text { tot }}}{\partial p_{b}}=-\frac{p_{b} M}{12 \lambda b}, \dot{\phi}=\frac{\partial \mathcal{H}_{\mathrm{EiBI}, \text { tot }}}{\partial p_{\phi}}=\frac{p_{\phi} N}{2 a^{3} l}, \\
\dot{p}_{b}= & -\frac{\partial \mathcal{H}_{\mathrm{EiBI}, \text { tot }}}{\partial b}=\frac{6 \lambda \dot{b}^{2}}{M}-\frac{1}{\kappa}\left[6 \lambda^{2} M b^{2}\right. \\
& \left.-\frac{3 \lambda b^{2} N^{2}}{M}-3 \lambda a^{2} M\right] \neq 0, \\
\dot{p}_{\phi}= & -\frac{\partial \mathcal{H}_{\mathrm{EiBI}, \text { tot }}}{\partial \phi}=-\frac{1}{\kappa}\left[2 \kappa N a^{3} V^{\prime}(\phi)\right] \\
= & -2 N a^{3} V^{\prime}(\phi) \neq 0, \\
\dot{p}_{a}= & -\frac{\partial \mathcal{H}_{\mathrm{EiBI}, \text { tot }}}{\partial a}=\frac{3 a^{2} l \dot{\phi}^{2}}{N}+\frac{1}{\kappa}\left[6 \lambda a b M-6 \kappa a^{2} N V(\phi)\right. \\
& \left.+6 \kappa a^{2} p_{m}(a) N\right]=0, \\
\dot{p}_{N}= & -\frac{\partial \mathcal{H}_{\mathrm{EiBI}, \text { tot }}}{\partial N}=-\frac{a^{3} l \dot{\phi}^{2}}{N^{2}}+\frac{1}{\kappa}\left[\frac{2 \lambda b^{3} N}{M}-2 \kappa a^{3} V(\phi)\right. \\
& \left.-2 \kappa \rho_{m}(a) a^{3}\right]=0, \\
\dot{p}_{M}= & -\frac{\partial \mathcal{H}_{\mathrm{EiBI}, \text { tot }}}{\partial M}=-\frac{6 \lambda b \dot{b}^{2}}{M^{2}} \\
& +\frac{1}{\kappa}\left[-2 \lambda^{2} b^{3}-\frac{\lambda b^{3} N^{2}}{M^{2}}+3 \lambda a^{2} b\right]=0 .
\end{aligned}
$$

For the EiBI Hamiltonian, it should be noted that $\dot{a}=$ $\lambda_{a}, \dot{N}=\lambda_{N}, \dot{M}=\lambda_{M}$ are the primary constraints and $\dot{p}_{a}=\dot{p}_{N}=\dot{p}_{M}=0$ are the secondary constraints that must be valid at all times [69] resulting in $p_{a}=p_{M}=p_{N}=0$. In order to obtain the dynamic solutions, we have to calculate the Euler-Lagrange equations for $a(t), b(t), M(t), N(t)$, and $\phi(t)$ as shown below:

$$
\begin{aligned}
\frac{3 a^{2} l \dot{\phi}^{2}}{N}+\frac{6 \lambda a b M}{\kappa}-6 N a^{2} V(\phi)+6 N a^{2} p_{m}(a) & =0, \\
\ddot{b}-\frac{\dot{b} \dot{M}}{M}+\frac{\dot{b}^{2}}{2 b}-\frac{\lambda M^{2} b}{2 \kappa}+\frac{b N^{2}}{4 \kappa}+\frac{a^{2} M^{2}}{4 \kappa b} & =0, \\
\frac{6 \lambda b \dot{b}^{2}}{M^{2}}-\frac{2 \lambda^{2} b^{3}}{\kappa}+\frac{3 \lambda a^{2} b}{\kappa}-\frac{\lambda b^{3}}{\kappa} \frac{N^{2}}{M^{2}} & =0, \\
-\frac{a^{3} l \dot{\phi}^{2}}{N^{2}}+\frac{2 \lambda b^{3} N}{\kappa M}-2 a^{3} V(\phi)-2 a^{3} \rho_{m}(a) & =0, \\
\ddot{\phi}+\left(3 \frac{\dot{a}}{a}+\frac{\dot{N}}{N}\right) \dot{\phi}+\frac{V^{\prime}(\phi) N^{2}}{l} & =0,
\end{aligned}
$$

where the conservation equation [68], $d \rho_{m} / d a=-3\left(\rho_{m}+\right.$ $\left.p_{m}\right) / a$, has been used to yield Eq. (3.7).

\section{Noether gauge symmetries in EiBI gravity}

Noether vector $\left(\mathrm{X}_{N G S}\right)$ and the first prolongation vector field $\left(\mathrm{X}_{\mathrm{NGS}}^{[1]}\right)$ related to EiBI Lagrangian, as shown in Eq. (2.7), can be constructed as follows:

$\mathrm{X}_{N G S}=\tau \frac{\partial}{\partial t}+\alpha \frac{\partial}{\partial a}+\beta \frac{\partial}{\partial b}+\gamma \frac{\partial}{\partial N}+\xi \frac{\partial}{\partial M}+\varphi \frac{\partial}{\partial \phi}$,

$\mathrm{X}_{\mathrm{NGS}}^{[1]}=\mathrm{X}_{\mathrm{NGS}}+\dot{\alpha} \frac{\partial}{\partial \dot{a}}+\dot{\beta} \frac{\partial}{\partial \dot{b}}+\dot{\gamma} \frac{\partial}{\partial \dot{N}}+\dot{\xi} \frac{\partial}{\partial \dot{M}}+\dot{\varphi} \frac{\partial}{\partial \dot{\phi}}$,

where the undetermined parameters $\left\{\tau\left(t, q^{i}\right), \alpha\left(t, q^{i}\right)\right.$, $\left.\beta\left(t, q^{i}\right), \gamma\left(t, q^{i}\right), \xi\left(t, q^{i}\right), \varphi\left(t, q^{i}\right)\right\}$ are possibly functioned by $\left\{t, q^{i}\right\}=\{t, a, b, N, M, \phi\}$. Their time derivative can be defined as

$\dot{\alpha}(t, a, b, N, M, \phi)=\mathrm{D}_{t} \alpha-\dot{a} \mathrm{D}_{t} \tau$,

for variable $\alpha(t)$. This can be applied in the same way for other undetermined variables. The operator of a total differentiation $\left(\mathrm{D}_{t}\right)$ with respect to $t$ in EiBI gravity can be defined as

$\mathrm{D}_{t}=\frac{\partial}{\partial t}+\dot{a} \frac{\partial}{\partial a}+\dot{b} \frac{\partial}{\partial b}+\dot{M} \frac{\partial}{\partial M}+\dot{N} \frac{\partial}{\partial N}+\dot{\phi} \frac{\partial}{\partial \phi}$.

It is worth noting that $\frac{\partial \mathcal{L}_{\text {EiBI }}}{\partial t}=0$ because there has no time variable $(t)$ expressed explicitly in $\mathcal{L}_{\mathrm{EiBI}}$. The vector field $\mathrm{X}_{N G S}^{[1]}$ is a NGS of a Lagrangian $\mathcal{L}(t, a, b, \phi, M, N, \dot{b}, \dot{\phi})$, if there exists a gauge function $\mathrm{B}(t, a, b, \phi, M, N)$ which obeys the following NGS condition (see Ref. [71] for explicit derivation):

$\mathrm{X}_{N G S}^{[1]} \mathcal{L}+\mathcal{L} \mathrm{D}_{t} \tau=\mathrm{D}_{t} \mathrm{~B}$.

For NSG without gauge term and the prolongation part of the vector field, i.e. $\mathrm{B}\left(t, q^{i}\right)=0$, it requires that $\tau\left(t, q^{i}\right)=0$. Accordingly, Eq. (4.5) can be reduced to $£_{X_{\mathrm{NGS}}} \mathcal{L}=0$ that is the condition for Noether symmetry [62]. After using the Noether gauge symmetries condition with the EiBI point-like Lagrangian, this provides us with eighty terms for $\mathrm{X}_{N G S}^{[1]} \mathcal{L}+$ $\mathcal{L} \mathrm{D}_{t} \tau=\mathrm{D}_{t} \mathrm{~B}$ as shown below:

$$
\begin{aligned}
& \frac{6 \lambda a b M \alpha}{\kappa}-\frac{6 \lambda a^{2} N \alpha}{\kappa}-6 a^{2} N \rho(a) \alpha-6 a^{2} N V(\phi) \alpha \\
& +\frac{3 \lambda a^{2} M \beta}{\kappa}-\frac{6 \lambda b^{2} M \beta}{\kappa}+\frac{3 \lambda b^{2} N^{2} \beta}{\kappa M}-\frac{2 \lambda a^{3} \gamma}{\kappa} \\
& +\frac{2 \lambda b^{3} N \gamma}{\kappa M}-2 a^{3} \rho(a) \gamma \\
& -2 a^{3} V(\phi) \gamma+\frac{3 \lambda a^{2} b \xi}{\kappa}-\frac{2 \lambda^{2} b^{3} \xi}{\kappa}-\frac{\lambda b^{3} N^{2} \xi}{\kappa M^{2}} \\
& -\frac{6 \lambda \beta \dot{b}^{2}}{M}+\frac{6 \lambda b \xi \dot{b}^{2}}{M^{2}}-2 a^{3} N \alpha \rho^{\prime}(a)
\end{aligned}
$$




$$
\begin{aligned}
& -2 a^{3} N \varphi V^{\prime}(\phi)+\frac{3 l a^{2} \alpha \dot{\phi}^{2}}{N}-\frac{l a^{3} \gamma \dot{\phi}^{2}}{N^{2}}-\frac{12 \lambda b \dot{b} \beta_{t}}{M} \\
& +\frac{3 \lambda a^{2} b M \tau_{t}}{\kappa}-\frac{2 \lambda^{2} b^{3} M \tau_{t}}{\kappa}-\frac{2 \lambda a^{3} N \tau_{t}}{\kappa}+\frac{\lambda b^{3} N^{2} \tau_{t}}{\kappa M} \\
& -2 a^{3} N \rho(a) \tau_{t}-2 a^{3} N V(\phi) \tau_{t} \\
& +\frac{6 \lambda b \dot{b}^{2} \tau_{t}}{M}-\frac{l a^{3} \dot{\phi}^{2} \tau_{t}}{N}+\frac{2 l a^{3} \dot{\phi} \varphi_{t}}{N} \\
& -\frac{24 \lambda b \dot{b} \dot{\phi} \beta_{\phi}}{M}+\frac{6 \lambda a^{2} b M \dot{\phi} \tau_{\phi}}{\kappa} \\
& -\frac{4 \lambda^{2} b^{3} M \dot{\phi} \tau_{\phi}}{\kappa}-\frac{4 \lambda a^{3} N \dot{\phi} \tau_{\phi}}{\kappa} \\
& +\frac{2 \lambda b^{3} N^{2} \dot{\phi} \tau_{\phi}}{\kappa M}-4 a^{3} N \rho(a) \dot{\phi} \tau_{\phi}-4 a^{3} N V(\phi) \dot{\phi} \tau_{\phi} \\
& +\frac{12 \lambda b \dot{b}^{2} \dot{\phi} \tau_{\phi}}{M}-\frac{2 l a^{3} \dot{\phi}^{3} \tau_{\phi}}{N}+\frac{4 l a^{3} \dot{\phi}^{2} \varphi_{\phi}}{N} \\
& -\frac{24 \lambda b \dot{b} \dot{M} \beta_{M}}{M}+\frac{6 \lambda a^{2} b M \dot{M} \tau_{M}}{\kappa} \\
& -\frac{4 \lambda^{2} b^{3} M \dot{M} \tau_{M}}{\kappa}-\frac{4 \lambda a^{3} N \dot{M} \tau_{M}}{\kappa}+\frac{2 \lambda b^{3} N^{2} \dot{M} \tau_{M}}{\kappa M} \\
& -4 a^{3} N \rho(a) \dot{M} \tau_{M}-4 a^{3} N V(\phi) \dot{M} \tau_{M}+\frac{12 \lambda b \dot{b}^{2} \dot{M} \tau_{M}}{M} \\
& -\frac{2 l a^{3} \dot{M} \dot{\phi}^{2} \tau_{M}}{N}+\frac{4 l a^{3} \dot{M} \dot{\phi} \varphi_{M}}{N}-\frac{24 \lambda b \dot{b} \dot{N} \beta_{N}}{M} \\
& +\frac{6 \lambda a^{2} b M \dot{N} \tau_{N}}{\kappa}-\frac{4 \lambda^{2} b^{3} M \dot{N} \tau_{N}}{\kappa}-\frac{4 \lambda a^{3} N \dot{N} \tau_{N}}{\kappa} \\
& +\frac{2 \lambda b^{3} N^{2} \dot{N} \tau_{N}}{\kappa M}-4 a^{3} N \rho(a) \dot{N} \tau_{N}-4 a^{3} N V(\phi) \dot{N} \tau_{N} \\
& +\frac{12 \lambda b \dot{b}^{2} \dot{N} \tau_{N}}{M}-\frac{2 l a^{3} \dot{N} \dot{\phi}^{2} \tau_{N}}{N}+\frac{4 l a^{3} \dot{N} \dot{\phi} \varphi_{N}}{N} \\
& -\frac{24 \lambda b \dot{b}^{2} \beta_{b}}{M}+\frac{6 \lambda a^{2} b M \dot{b} \tau_{b}}{\kappa}-\frac{4 \lambda^{2} b^{3} M \dot{b} \tau_{b}}{\kappa} \\
& -\frac{4 \lambda a^{3} N \dot{b} \tau_{b}}{\kappa}+\frac{2 \lambda b^{3} N^{2} \dot{b} \tau_{b}}{\kappa M}-4 a^{3} N \rho(a) \dot{b} \tau_{b} \\
& -4 a^{3} N V(\phi) \dot{b} \tau_{b}+\frac{12 \lambda b \dot{b}^{3} \tau_{b}}{M}-\frac{2 l a^{3} \dot{b} \dot{\phi}^{2} \tau_{b}}{N} \\
& +\frac{4 l a^{3} \dot{b} \dot{\phi} \varphi_{b}}{N}-\frac{24 \lambda b \dot{a} \dot{b} \beta_{a}}{M}+\frac{6 \lambda a^{2} b M \dot{a} \tau_{a}}{\kappa} \\
& -\frac{4 \lambda^{2} b^{3} M \dot{a} \tau_{a}}{\kappa}-\frac{4 \lambda a^{3} N \dot{a} \tau_{a}}{\kappa}+\frac{2 \lambda b^{3} N^{2} \dot{a} \tau_{a}}{\kappa M} \\
& -4 a^{3} N \rho(a) \dot{a} \tau_{a}-4 a^{3} N V(\phi) \dot{a} \tau_{a}+\frac{12 \lambda b \dot{a} \dot{b}^{2} \tau_{a}}{M} \\
& -\frac{2 l a^{3} \dot{a} \dot{\phi}^{2} \tau_{a}}{N}+\frac{4 l a^{3} \dot{a} \dot{\phi} \varphi_{a}}{N}=B_{t}+\dot{a} B_{a}+\dot{b} B_{b} \\
& +\dot{M} B_{M}+\dot{N} B_{N}+\dot{\phi} B_{\phi} \text {. }
\end{aligned}
$$

If the Noether symmetry condition $£_{\mathrm{X}_{\mathrm{NGS}}} \mathcal{L}_{\mathrm{EiBI}}=0$ is satisfied, then the function $\Sigma_{0}=\alpha^{i} \frac{\partial \mathcal{L}}{\partial \dot{q}^{i}}$ is a constant of motion [72]. This gives

$$
\begin{aligned}
\Sigma_{0, \mathrm{EiBI}}= & \alpha^{i} \frac{\partial \mathcal{L}}{\partial \dot{q}^{i}}=\beta \frac{\partial \mathcal{L}}{\partial \dot{b}}+\varphi \frac{\partial \mathcal{L}}{\partial \dot{\phi}}=\beta\left[-\frac{12 \lambda b \dot{b}}{M}\right] \\
& +\varphi\left[\frac{2 a^{3} l \dot{\phi}}{N}\right]
\end{aligned}
$$

where two unknown functions $\beta$ and $\varphi$ will be studied in the next section. Up to this point, it is worth mentioning a dimension analysis of each variable, i.e. [dimensionless] $=$ $[\lambda]=[l]=[\mathrm{a}]=[\mathrm{b}]=[\phi]=[N]=[M] ; \quad[\alpha]=[\beta]=$ $[\gamma]=[\xi]=[\varphi]=[\tau]=\left[M_{P}^{-1}\right] ;[\kappa]=\left[M_{P}^{-2}\right] ; \quad[\dot{a}]=$ $[\dot{b}]=[\dot{\phi}]=\left[M_{p}\right] ; \quad\left[\varphi_{t}\right]=\left[\beta_{t}\right]=\left[\tau_{t}\right]=\left[M_{P}^{-2}\right]$.

\section{Remarks on exact cosmological solutions}

After a separation of monomials, we can quantify the system equations to yield

$$
\begin{aligned}
\tau_{a}= & \tau_{b}=\tau_{M}=\tau_{N}=\tau_{\phi}=0 \\
\beta_{a}= & \beta_{M}=\beta_{N}=0 \\
\varphi_{a}= & \varphi_{M}=\varphi_{N}=0 \\
0= & -\frac{6 \lambda b \beta_{\phi}}{M}+\frac{l a^{3} \varphi_{b}}{N} \\
0= & 3 \alpha-\frac{a \gamma}{N}-a \tau_{t}+4 a \varphi_{\phi} \\
0= & -\beta+\frac{b \xi}{M}+b \tau_{t}-4 b \beta_{b} \\
B_{a}= & B_{M}=B_{N}=0 \\
B_{b}= & -\frac{12 \lambda b \beta_{t}}{M} \\
B_{\phi}= & \frac{2 l a^{3} \varphi_{t}}{N} \\
B_{t}= & \alpha\left[\frac{6 \lambda a b M}{\kappa}-\frac{6 \lambda a^{2} N}{\kappa}-6 a^{2} N \rho(a)-6 a^{2} N V(\phi)\right. \\
& \left.-2 a^{3} N \rho^{\prime}(a)\right]+\beta\left[\frac{3 \lambda a^{2} M}{\kappa}-\frac{6 \lambda^{2} b^{2} M}{\kappa}+\frac{3 \lambda b^{2} N^{2}}{\kappa M}\right] \\
& +\gamma\left[-\frac{2 \lambda a^{3}}{\kappa}+\frac{2 \lambda b^{3} N}{\kappa M}-2 a^{3} \rho(a)-2 a^{3} V(\phi)\right] \\
& +\xi\left[\frac{3 \lambda a^{2} b}{\kappa}-\frac{2 \lambda^{2} b^{3}}{\kappa}-\frac{\lambda b^{3} N^{2}}{\kappa M^{2}}\right]+\varphi\left[-2 a^{3} N V^{\prime}(\phi)\right] \\
& +\tau_{t}\left[\frac{3 \lambda a^{2} b M}{\kappa}-\frac{2 \lambda^{2} b^{3} M}{\kappa}-\frac{2 \lambda a^{3} N}{\kappa}+\frac{\lambda b^{3} N^{2}}{\kappa M}\right. \\
& \left.-2 a^{3} N \rho(a)-2 a^{3} N V(\phi)\right]
\end{aligned}
$$

From Eqs. (5.2), (5.3) and (5.4), one found that $\beta=\beta(b)$ and $\varphi(\phi)$. If we choose

$$
\begin{aligned}
& \beta(b)=c_{1} b, \\
& \varphi(\phi)=c_{2} \phi .
\end{aligned}
$$

From Eq. (5.1), there is only one possibility left with $\tau_{t} \neq 0$. Therefore the polynomial of $\alpha, \beta, \varphi, \gamma$, and $\xi$ no longer have 
hold in $\tau(t)$. Proposing the linearity of the relations expressed in Eqs. (5.5) and (5.6), we have to set

$$
\begin{aligned}
\alpha(a) & =c_{3} a, \\
\gamma(N) & =c_{4} N, \\
\xi(M) & =c_{5} M .
\end{aligned}
$$

With this setting, we can solve Eqs. (5.5) and (5.6) to get

$$
\begin{aligned}
& \tau(t)=\left(3 c_{3}-c_{4}+4 c_{2}\right) t+c_{6}, \\
& \tau(t)=\left(5 c_{1}-c_{5}\right) t+c_{7} .
\end{aligned}
$$

In order to write $\tau(t)$ in a single form, we have to set $c_{6}=c_{7}$ and $3 c_{3}-c_{4}+4 c_{2}=5 c_{1}-c_{5}$. There are three equations contributed of gauge function,

$$
\begin{aligned}
B_{b}= & -\frac{12 \lambda b \beta_{t}}{M}=-\frac{12 c_{1} \lambda b \dot{b}}{M}, \\
B_{\phi}= & \frac{2 l a^{3} \varphi_{t}}{N}=\frac{2 c_{2} l a^{3} \dot{\phi}}{N}, \\
B_{t}= & c_{3}\left[\frac{6 \lambda a^{2} b M}{\kappa}-\frac{6 \lambda a^{3} N}{\kappa}-6 a^{3} N \rho(a)-6 a^{3} N V(\phi)\right. \\
& \left.-2 a^{3} N \rho^{\prime}(a)\right]+c_{1}\left[\frac{3 \lambda a^{2} b M}{\kappa}-\frac{6 \lambda^{2} b^{3} M}{\kappa}+\frac{3 \lambda b^{3} N^{2}}{\kappa M}\right] \\
& +c_{4}\left[-\frac{2 \lambda a^{3} N}{\kappa}+\frac{2 \lambda b^{3} N^{2}}{\kappa M}-2 a^{3} N \rho(a)-2 a^{3} N V(\phi)\right] \\
& +c_{5}\left[\frac{3 \lambda a^{2} b M}{\kappa}-\frac{2 \lambda^{2} b^{3} M}{\kappa}-\frac{\lambda b^{3} N^{2}}{\kappa M}\right] \\
& +\left(3 c_{3}-c_{4}+4 c_{2}\right)\left[\frac{3 \lambda a^{2} b M}{\kappa}-\frac{2 \lambda^{2} b^{3} M}{\kappa}-\frac{2 \lambda a^{3} N}{\kappa}\right. \\
& \left.+\frac{\lambda b^{3} N^{2}}{\kappa M}-2 a^{3} N \rho(a)-2 a^{3} N V(\phi)\right]-2 c_{2} a^{3} N \phi V^{\prime}(\phi),
\end{aligned}
$$

where we use $\beta_{t}=\frac{\partial \beta}{\partial b} \frac{d b}{d t}=c_{1} \dot{b}$ and $\varphi_{t}=\frac{\partial \varphi}{\partial \phi} \frac{d \phi}{d t}=c_{2} \dot{\phi}$ to get Eqs. (5.18) and (5.19). From Eqs. (5.4), (5.9) and (5.8), it is easy to see that

$\frac{6 \lambda b}{M}=-\frac{B_{b}}{2 \beta_{t}}, \quad \frac{l a^{3}}{N}=\frac{B_{\phi}}{2 \varphi_{t}}$.

This gives the following relation,

$$
\begin{aligned}
\frac{6 \lambda b \beta_{\phi}}{M} & =\frac{l a^{3} \varphi_{b}}{N}, \\
-\frac{B_{b}}{2 \beta_{t}} \beta_{\phi} & =\frac{B_{\phi}}{2 \varphi_{t}} \varphi_{b},
\end{aligned}
$$

This confirms again that $\beta_{\phi}=\varphi_{b}=0$, but keeps $\beta_{t} \neq 0$ and $\varphi_{t} \neq 0$. That also means that $B_{b} \neq 0$ and $B_{\phi} \neq 0$. The boundary term can be also partly derived from Eqs. (5.18) and (5.19), that is

$B_{(b, \phi)}=-\frac{6 c_{1} \lambda b^{2} \dot{b}}{M}+\frac{2 c_{2} a^{3} l \phi \dot{\phi}}{N}$.

It is worth noting that $c_{1}$ is just an arbitrary constant and we can redefine it by replacing $c_{1} \rightarrow 2 c_{1}$. Interestingly, this is exactly matched with the constant of motions of EiBI gravity by this setting.

$\Sigma_{0, \mathrm{EiBI}}=-\frac{12 c_{1} \lambda b^{2} \dot{b}}{M}+\frac{2 c_{2} a^{3} l \phi \dot{\phi}}{N}$.

We hence expect that the rest of the expression for boundary term, i.e. $-\frac{12 c_{1} \lambda b \dot{b}}{M}$, may relate with $B_{t}$. The relation between the the constant of motion and the boundary term has shown in [71]. Clearly, Eq. (5.20) can be rewritten as

$$
\begin{aligned}
B_{t}= & \frac{\lambda a^{3} N}{\kappa}\left[-12 c_{3}-8 c_{2}\right]+a^{3} N \rho(a)\left[-12 c_{3}-8 c_{2}\right] \\
& +a^{2} N V(\phi)\left[-12 c_{3}-8 c_{2}\right] \\
& +\frac{\lambda a^{2} b M}{\kappa}\left[3 c_{1}+12 c_{2}+15 c_{3}-3 c_{4}+3 c_{5}\right] \\
& +\frac{\lambda^{2} b^{3} M}{\kappa}\left[-6 c_{1}-8 c_{2}-6 c_{3}+2 c_{4}-2 c_{5}\right] \\
& +\frac{\lambda b^{3} N^{2}}{\kappa M}\left[3 c_{1}+4 c_{2}+3 c_{3}+c_{4}-c_{5}\right] \\
& -2 a^{3} N\left[c_{3} \rho^{\prime}(a)+c_{2} V^{\prime}(\phi)\right],
\end{aligned}
$$

where it is very plausible to set $c_{4}=c_{5}$ and $c_{3}=-\frac{2}{3} c_{2}$. Notice that some terms of $B_{t}$ become zero using simple algebra, i.e.

$$
\begin{aligned}
B_{t}= & \frac{\lambda a^{2} b M}{\kappa}\left[3 c_{1}+2 c_{2}\right]+\frac{\lambda^{2} b^{3} M}{\kappa}\left[3 c_{1}+2 c_{2}\right] \\
& +\frac{\lambda b^{3} N^{2}}{\kappa M}\left[3 c_{1}+2 c_{2}\right] \\
& -2 c_{2} a^{3} N\left[-\frac{2}{3} \rho^{\prime}(a)+V^{\prime}(\phi)\right] .
\end{aligned}
$$

If we further set $c_{1}=-\frac{2}{3} c_{2}=-\frac{2}{3}\left(-\frac{3}{2}\right) c_{3}=c_{3}$, it is worth seeing that

$$
\begin{aligned}
B_{t} & =c_{1} a^{2} N\left[-2 \rho^{\prime}(a)+3 V^{\prime}(\phi)\right], \\
& =6 c_{1} a^{2} N\left[\frac{\rho_{m}(a)+P_{m}(a)}{a}+V^{\prime}(\phi)\right],
\end{aligned}
$$

where the continuity equation, $\rho^{\prime}(a)=-3\left(\frac{\left(\rho_{m}+P_{m}\right)}{a}\right)$, has been used to obtain an expression in Eq. (5.28). Due to the appearance of $c_{1}$ on the right-hand side of Eq. (5.28), it is reasonable to set $B_{t} \equiv-\frac{12 c_{1} \lambda b \dot{b}}{M}$. This gives the relation between two scale factors, $b(t)$ and $a(t)$, as shown below

$b \dot{b}=-\frac{3 a^{2} N(t) M(t)}{1+\kappa \Lambda}\left[\frac{\rho_{m}(a)+P_{m}(a)}{a}+V^{\prime}(\phi)\right]$.

To explain the expanding phase of the universe at late time, the exponential potential, i.e. $V(\phi)=V_{0} e^{-\phi}$ is more suitable 
for the model of gravity than the power law potential, $V(\phi)=$ $V_{0} \phi^{2}$. For the exponential potential, this gives

$b \dot{b}=-\frac{3 a^{2} N(t) M(t)}{1+\kappa \Lambda}\left[\frac{\rho_{m}(a)+P_{m}(a)}{a}-V_{0} e^{-\phi}\right]>0$.

whereas

$b \dot{b}=-\frac{3 a^{2} N(t) M(t)}{1+\kappa \Lambda}\left[\frac{\rho_{m}(a)+P_{m}(a)}{a}+2 V_{0} \phi(t)\right]<0$.

for the power laws potential. Here we are interested studying a case when $V_{0} e^{-\phi} \simeq V_{0}(1-\phi(t))$ as an example where $\phi(t) \ll 1$ and using the limit ranged of $\kappa \Lambda$ as $1.12 \times 10^{-4} \lesssim$ $\kappa \Lambda \lesssim 2.10 \times 10^{-3}$ and $M=\sqrt{1+\kappa \Lambda}, N(t)=1, \frac{a^{2}}{b^{2}}=$ $\frac{1}{1+\kappa \Lambda}$ as given in [73]. It was noticed that the constraints on the free parameter of EiBI theories leading to the strongest constraints available in Refs. [74,75]. In the present work, this gives the de Sitter solution in EiBI gravity model based on the Noether gauge symmetry,

$\frac{\dot{b}}{b} \simeq \frac{3 V_{0}}{\sqrt{1+\kappa \Lambda}}, \quad b(t)=e^{\frac{3 V_{0} t}{\sqrt{1+\kappa \Lambda}}}$.

Clearly from Eq. (5.30), if there is no contribution from the matter fields, i.e. $\rho_{m}=0$, the scalar field $\phi(t) \rightarrow 0$, yielding $H_{b}=\frac{\dot{b}}{b} \rightarrow$ const. This is the de Sitter phase of EiBI Universe. There were previous works by Avelino with weaker constraints from astrophysical and nuclear physics scenarios as well $[76,77]$.

\section{Conclusion}

We revisited a formal framework of the Eddington-inspired Born-Infeld (EiBI) theory of gravity and derived the pointlike Lagrangian for underlying theory based on the use of Noether gauge symmetries (NGS). A Hessian matrix and quantify Euler-Lagrange equations of EiBI universe have been explicitly quantified. We also discussed the NGS approach for the Eddington-inspired Born-Infeld theory and comment on exact cosmological solutions.

We end this work by providing some remarks. As expected, the NGS method can simplify the complication of constraint equations and also helps us to simplify further the gauge function equations with the linear forms of $\beta(b), \varphi(\phi), \alpha(a), \gamma(N), \xi(M)$ and $\tau(t)$. By assuming the equality of $B_{t}$ and the constant of motion, the two scale factors $a(t)$ and $b(t)$ are correlated through the matter fields and the scalar field. Interestingly, we show that there exists the de Sitter solution in this gravity model.

Acknowledgements T. Kanesom is financially supported by the Science Achievement Scholarship of Thailand (SAST) and Graduate School and the Department of Physics, Prince of Songkla University. P. Channuie acknowledged the Mid-Cereer Research Grant 2020 from National Research Council of Thailand under a contract No. NFS6400117.

Data Availability Statement This manuscript has no associated data or the data will not be deposited. [Authors' comment: We do not have any data related to this manuscript. It is a theoretical work only and no data is involved.]

Open Access This article is licensed under a Creative Commons Attribution 4.0 International License, which permits use, sharing, adaptation, distribution and reproduction in any medium or format, as long as you give appropriate credit to the original author(s) and the source, provide a link to the Creative Commons licence, and indicate if changes were made. The images or other third party material in this article are included in the article's Creative Commons licence, unless indicated otherwise in a credit line to the material. If material is not included in the article's Creative Commons licence and your intended use is not permitted by statutory regulation or exceeds the permitted use, you will need to obtain permission directly from the copyright holder. To view a copy of this licence, visit http://creativecomm ons.org/licenses/by/4.0/.

Funded by $\mathrm{SCOAP}^{3}$.

\section{References}

1. S. Perlmutter et al., Astrophys. J. 517, 565 (1999)

2. A.G. Riess et al., Astron. J. 116, 1009 (1998)

3. P.A.R. Ade et al., Astron. Astrophys. 594, A13 (2016)

4. P.A.R. Ade et al. (Planck Collaboration), Astron. Astrophys. 594, A20 (2016)

5. P.A.R. Ade et al., (BICEP2 Collaboration) Phys. Rev. Lett. 112, (2014)

6. P.A.R. Ade et al., Phys. Rev. Lett. 114, (2015)

7. P.A.R. Ade et al., Phys. Rev. Lett. 116, (2016)

8. E. Komatsu et al. [WMAP Collaboration], Astrophys. J. Suppl. 192, 18 (2011) ibid

9. G. Hinshaw et al. (WMAP Collaboration), Astrophys. J. Suppl. 208, 19 (2013)

10. M. Tegmark et al. (SDSS Collaboration), Phys. Rev. D 69, 103501 (2004) U. Seljak et al. [SDSS Collaboration], Phys. Rev. D 71, 103515 (2005)

11. U. Seljak et al. (SDSS Collaboration), Phys. Rev. D 71, 103515 (2005)

12. D.J. Eisenstein et al., SDSS Collaboration. Astrophys. J. 633, 560 (2005)

13. B. Jain, A. Taylor, Phys. Rev. Lett. 91, (2003)

14. Y. Akrami et al. Planck (2018). arXiv:1807.06205 [astro-ph]

15. S. Weinberg, (2000). arXiv:astro-ph/0005265

16. H. Velten, R. vom Marttens, W. Zimdahl, Eur. Phys. J. C 74(11), 3160 (2014)

17. S. Nojiri, S.D. Odintsov, Phys. Rept. 505, 59 (2011)

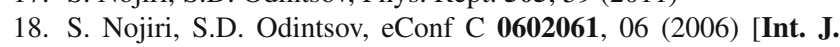
Geom. Meth. Mod. Phys. 4, 115 (2007)]

19. S. Capozziello, V. Faraoni, Beyond Einstein Gravity (Springer, Dordrecht, 2010)

20. S. Capozziello, M. De Laurentis, Phys. Rept. 509, 167 (2011)

21. K. Bamba, S.D. Odintsov, Symmetry 7(1), 220 (2015)

22. E.J. Copeland, M. Sami, S. Tsujikawa, Int. J. Mod. Phys. D 15, $1753(2006)$

23. P.G. Bergmann, Int. J. Theor. Phys. 1, 25 (1968)

24. H.A. Buchdahl, Mon. Not. R. Astron. Soc. 150, 1 (1970)

25. T.P. Sotiriou, V. Faraoni, Rev. Mod. Phys. 82, 451 (2010)

26. A. De Felice, S. Tsujikawa, Living Rev. Rel. 13, 3 (2010) 
27. J. Beltran Jimenez, L. Heisenberg, G.J. Olmo, D. Rubiera-Garcia, Phys. Rept. 727, 1-129 (2018)

28. S. Nojiri, S.D. Odintsov, V.K. Oikonomou, Late-time evolution. Phys. Rept. 692, 1 (2017)

29. A. Delhom, G.J. Olmo, E. Orazi, JHEP 11, 149 (2019). https://doi. org/10.1007/JHEP11(2019)149. arXiv:1907.04183 [gr-qc]

30. V.I. Afonso, G.J. Olmo, E. Orazi, D. Rubiera-Garcia, Eur. Phys. J. C 78(10), 866 (2018)

31. V.I. Afonso, G.J. Olmo, D. Rubiera-Garcia, Phys. Rev. D 97(2), (2018)

32. J. Beltran Jimenez, L. Heisenberg, G.J. Olmo, D. Rubiera-Garcia, JCAP 10, 029 (2017). https://doi.org/10.1088/1475-7516/2017/ 10/029. arXiv:1707.08953 [hep-th] [erratum: JCAP 08 (2018), E01]

33. G.J. Olmo, E. Orazi, D. Rubiera-Garcia, Eur. Phys. J. C 80(11), 1018 (2020)

34. M. Guerrero, G. Mora-Pérez, G.J. Olmo, E. Orazi, D. RubieraGarcia, JCAP 07, 058 (2020)

35. J.R. Nascimento, G.J. Olmo, P.J. Porfírio, A.Y. Petrov, A.R. Soares, Phys. Rev. D 101(6), (2020)

36. V.I. Afonso, G.J. Olmo, E. Orazi, D. Rubiera-Garcia, JCAP 12, 044 (2019)

37. K F. Dialektopoulos, S. Capozziello, Int. J. Geom. Meth. Mod. Phys. 15(supp.01), 1840007 (2018)

38. S. Capozziello, R. De Ritis, P. Scudellaro, Nuovo Cim. B 109, 159-165 (1994)

39. S. Capozziello, V. Faraon, Fundamental Theories of Physics, vol. 170 (Springer, Berlin, 2010)

40. P. Channuie, D. Momeni, Nucl. Phys. B 935, 256-270 (2018)

41. S. Capozziello, A. De Felice, JCAP 0808, 016 (2008)

42. N. Kaewkhao, T. Kanesom, P. Channuie, Nucl. Phys. B 931, 216225 (2018)

43. A.N. Nurbaki, S. Capozziello, C. Deliduman, Eur. Phys. J. C 80(2), $108(2020)$

44. F. Bajardi, S. Capozziello, Eur. Phys. J. C 80(8), 704 (2020)

45. F. Bajardi, S. Capozziello, D. Vernieri, Eur. Phys. J. Plus 135(12), $942(2020)$

46. S. Capozziello, R. de Ritis, Phys. Lett. A 177, 1 (1993)

47. D. Momeni, R. Myrzakulov, E. Güdekli, Int. J. Geom. Meth. Mod. Phys. 12(10), 1550101 (2015)

48. D. Momeni, R. Myrzakulov, Can. J. Phys. 94(8), 763 (2016)

49. A. Aslam, M. Jamil, D. Momeni, R. Myrzakulov, Can. J. Phys. 91, $93(2013)$

50. M. Jamil, D. Momeni, R. Myrzakulov, Eur. Phys. J. C 72, 2137 (2012)
51. S. Bahamonde, S. Capozziello, K.F. Dialektopoulos, Eur. Phys. J. C 77(11), 722 (2017)

52. M. Jamil, F. Mahomed, D. Momeni, Phys. Lett. B 702, 315-319 (2011)

53. S. Basilakos, M. Tsamparlis, A. Paliathanasis, Phys. Rev. D 83, (2011)

54. M. Tsamparlis, A. Paliathanasis, Gen. Rel. Grav. 43, 1861-1881 (2011)

55. M. Tsamparlis, A. Paliathanasis, Symmetry 10(7), 233 (2018)

56. S. Capozziello, G. Marmo, C. Rubano, P. Scudellaro, Int. J. Mod. Phys. D 6, 491 (1997)

57. U. Camci, Y. Kucukakca, 3 Phys. Rev. D 76, (2007)

58. M. Jamil, S. Ali, D. Momeni, R. Myrzakulov, Eur. Phys. J. C 72, 1998 (2012)

59. P. Channuie, D. Momeni, M.A. Ajmi, Eur. Phys. J. C 78(7), 588 (2018)

60. A. Aslam, M. Jamil, D. Momeni, R. Myrzakulov, M.A. Rashid, M. Raza, Astrophys. Space Sci. 348, 533 (2013)

61. I. Hussain, M. Jamil, F.M. Mahomed, Astrophys. Space Sci. 337, 373 (2012)

62. Y. Kucuakca, U. Camci, Astrophys. Space Sci. 338, 211 (2012)

63. T. Delsate, J. Steinhoff, Phys. Rev. Lett. 109, (2008)

64. M. Banados, P.G. Ferreira, Phys. Rev. Lett. 105, (2010)

65. J. Beltrán Jiménez, A. Delhom, Eur. Phys. J. C 79(8), 656 (2019)

66. J.B. Jiménez, A. Delhom, Eur. Phys. J. C 80(6), 585 (2020)

67. N. Rosen, Gen. Relativ. Gravit. 4, 435-447 (1973)

68. M. Bouhmadi-López, C. Chen, JCAP 11, 023 (2016)

69. M. Bojowald, Canonical Gravity and Application: Cosmology, Black Holes, and Quantum Gravity (Cambridge University Press, New York, 2011)

70. M. Henneaux, C. Teitelboim, Quantization of Gauge Systems (Princeton, Princeton University Press, 1992), pp. 4-5

71. K. Sundermeyer, Fundam. Theor. Phys. 176 (2014)

72. S. Capozziello, R. de Ritis, C. Rubano et al., Riv. Nuovo Cim. 19, 1-114 (1996)

73. N. Kaewkhao, Phys. Dark Univ. 27, (2020)

74. A. Delhom, V. Miralles, A. Peñuelas, Eur. Phys. J. C 80(4), 340 (2020)

75. A.D.I. Latorre, G.J. Olmo, M. Ronco, Phys. Lett. B 780, 294-299 (2018)

76. P.P. Avelino, JCAP 11, 022 (2012)

77. P.P. Avelino, Phys. Rev. D 85, (2012) 